\title{
Frequência cardíaca, perceção subjetiva de esforço e lactato sanguíneo nas aulas de jump fit e hidro jump
}

\author{
Heart rate, rating of perceived exertion and blood lactate levels in jump \\ fit and hydro jump classes
}

\author{
H.B. Moraes, R.G. Vale, A.L. Gomes, G.S. Novaes, J.V. Alves, D.A. Marinho, J.S. Novaes \\ ARTIGO ORIGINAL | ORIGINAL ARTICLE
}

\begin{abstract}
O objetivo deste estudo foi comparar as respostas da frequência cardíaca (FC), perceção subjetiva do esforço (PSE) e da concentração de lactato (LA) nas aulas de jump fit e hidro jump. A amostra foi constituída por 8 mulheres $(43.5 \pm 5.2$ anos, $160.0 \pm 4.68 \mathrm{~cm}, 58.93 \pm 4.15 \mathrm{~kg}$ e $25.69 \pm 4.40 \% \mathrm{MG})$, todas elas praticantes das aulas de fit jump. O estudo envolveu três momentos de avaliação. Na primeira foram avaliadas as medidas antropométricas, FC e LA em repouso, bem como a familiarização e treino da aula do hidro jump e da Escala de BORG. No segundo e terceiro momento de avaliação foram recolhidas as variáveis durante o hidro jump e jump fit, com 48 horas de intervalo entre as avaliações. Foi realizada a estatística descritiva e a inferencial através da ANOVA one-way com o Post Hoc de Tukey para medir a FC e LA e o teste de Friedman seguido do teste de Wilcoxon para a PSE. O estudo admitiu o nível de significância de $p<.05$. Não houve diferenças estatisticamente significativas entre os valores médios de todas as variáveis estudadas. O jump fit produziu efeitos semelhantes nas variáveis estudadas no meio terrestre e aquático.
\end{abstract}

Palavras-chave: minitrampolim, frequência cardíaca, perceção subjetiva de esforço e lactato sanguíneo

ABSTRACT

The purpose of the present study was to compare the heart rate responses (HR), rating of perceived exertion (RPE) and blood lactate levels in jump fit and hydro jump classes. The sample comprised 8 female subjects $(43.5 \pm 5.2$ years, $160.0 \pm 4.68 \mathrm{~cm}, 58.93 \pm 4.15 \mathrm{~kg}$ and $25.69 \pm 4.40 \% \mathrm{BF})$, all of them participants of jump fit classes. The study involved three evaluation moments. The first moment consisted of evaluating anthropometric measurements, resting heart rate and resting blood lactate levels, familiarization and training of hydro jump class and how to use the rating of perceived exertion scale (RPE). The second and third moments included collecting the variables during hydro jump class. 48 hours later, the variables during jump fit class were collected. Descriptive and inferential analysis consisted in applying one way ANOVA and Tukey's Post Hoc test in order to compare the HR and lactate blood levels during exercise, and further the Wilcoxon test to compare the RPE. A statistical significance was assumed as $p<.05$. No statistically relevant differences were found among the average values of all the variables studied. Jump classes had similar effects on the variables both in the terrestrial and aquatic context.

Keywords: rebound exercise, heart rate, rate of perceived exercitation (RPE) and blood lactate

Submetido: 24.04.2010 | Aceite: 20.04.2011

Hérida B. Moraes. Universidade Castelo Branco - Rio de Janeiro, Brasil.

Rodrigo G. Vale e André L. Gomes. Universidade Estácio de Sá - Rio de Janeiro, Brasil.

Giovanni S. Novaes. Universidade de Trás-os-Montes e Alto Douro - Vila Real, Portugal.

José Vilaça Alves. Centro de Investigação em Desporto, Saúde e Desenvolvimento Humano; Universidade de Trás-os-Montes e Alto Douro - Vila Real, Portugal.

Daniel Almeida Marinho. Centro de Investigação em Desporto, Saúde e Desenvolvimento Humano; Universidade da Beira Interior - Covilhã, Portugal.

Jefferson S. Novaes. Universidade Federal do Rio de Janeiro - Rio de Janeiro, Brasil.

Endereço para correspondência: Jefferson S. Novaes, Escola de Educação Física e Desportos, Univ. Federal do Rio de Janeiro, Av. Carlos Chagas Filho, 540, Cidade Universitária, CEP: 21941-599 Rio de Janeiro, RJ - Brasil

E-mail: jsnovaes@terra.com.br 
As estratégias utilizadas pelas academias para proporcionar aos seus alunos motivação e adesão verificam-se na variação das atividades físicas e no emprego de diferentes métodos de aulas. Estes visam atender os âmbitos das necessidades de seus praticantes, como por exemplo, a estética e a saúde (Mello, Boscolo, Esteves, \& Tukik, 2005). Dos diferentes tipos de métodos de aula de atividades de academia da atualidade, dois dos mais recentes são o jump fit e o hidro jump.

O jump fit pratica-se em cima de um minitrampolim no meio terrestre. Tem como objetivo a melhoria do condicionamento físico, proporcionando alterações fisiológicas, do tipo cardiovasculares e neuromusculares. Sua proposta possibilita incrementos em ambas as funções, através da estimulação das variáveis hemodinâmicas e metabólicas, por meio das ações musculares, de resistência e força, dos membros inferiores e abdómen (Alonso, Anjos, Leite, Gonçalves, \& Padovani, 2005; Anjos, Leite, Alonso, Gonçalves, \& Padovani, 2006; Freire \& Novaes, 2005; Perantoni, Derez, Lauria, Lima, \& Novaes, 2009; Smith, \& Bishop, 1988). Por sua vez, o hidro jump é um método que tem os mesmos objetivos e proposta metodológica do jump fit, porém com algumas modificações nos exercícios, devido à adaptação ao meio aquático (Anjos et al., 2006; Anjos, Leite, Alonso, Gonçalves, \& Padovani, 2007).

A prática de uma mesma metodologia de treino dentro e fora da água pode ter efeitos agudos e crónicos distintos. No meio aquático, os indicadores fisiológicos têm sido estudados (Graef \& Kruel, 2006). Entretanto, ao se fazer a comparação com o meio terrestre verifica-se que uma mesma atividade apresenta respostas diferenciadas (Svedenhag, \& Seger, 1992). Isto pode acontecer em virtude das propriedades físicas do meio líquido e terrestre se comportarem de forma diferente, tendo respostas fisiológicas, metabólicas e biomecânicas distintas dos indivíduos durante os exercícios (Kruel, 2000). A imersão, a temperatura da água e as diferentes posições corporais adota- das podem afetar o comportamento dos indicadores de intensidade do esforço durante a execução dos exercícios (Kruel, Tartaruga, Dias, Silva, Picanço, \& Rangel, 2005) ou mesmo durante a sua recuperação (Di Masi, Vale, Dantas, Barreto, Novaes, \& Reis, 2007).

Segundo Furtado, Simão e Lemos (2004), e Vendrusculo, Tartaruga, Coertjens, Pantoja, Petkowicz e Kruel (2004), a frequência cardíaca, a perceção subjetiva do esforço e a concentração de lactato são indicadores fisiológicos que podem ser utilizados como marcadores das alterações orgânicas sofridas pelo corpo, devido a este ter sido submetido ao stress e ao esforço, durante a prática do jump. Os estudos de Furtado et al. (2004) e Anjos et al. (2006) verificaram as respostas fisiológicas durante a prática do jump fit, resultando numa melhoria na capacidade cardiorrespiratória. Outros estudos confirmaram a hipótese de que uma sessão de jump pode influenciar negativamente o desempenho de um exercício de força (Lemos, Simão, Miranda, \& Novaes, 2007) ou de uma sequência de exercícios de força (Lemos, Simão, Miranda, \& Novaes, 2008). Algumas pesquisas observaram que a prática do hidro jump também aumenta a capacidade cardiorrespiratória (Anjos et al., 2006) e promovia alterações das variáveis neuromusculares (Anjos et al., 2006; Alonso et al., 2007). Entretanto, revendo a literatura, verificou-se a ausência de estudos que fizessem a comparação dos indicadores fisiológico (LA) e psicofisiológico (PSE) entre as atividades de jump dentro e fora da água.

Assim sendo, este estudo teve como objetivo comparar os efeitos agudos da prática do jump na frequência cardíaca (FC), nos níveis de lactato sanguíneo (LA) e na perceção subjetiva de esforço (PSE) num protocolo de aula igual tanto para meio aquático, como para meio terrestre.

\section{MÉTODO}

\section{Amostra}

A amostra não probabilística foi composta por 8 indivíduos do sexo feminino com valores 
médios de $43.5 \pm 5.2$ anos, $160.0 \pm 4.68 \mathrm{~cm}$, $58.93 \pm 4.15 \mathrm{~kg}$ e $25.69 \pm 4.40 \% \mathrm{MG}$, para a idade, estatura, massa corporal e percentagem de massa gorda estimada respetivamente, sendo todas saudáveis, fisicamente ativas e praticantes de jump fit. Para a seleção dos sujeitos respeitaram-se os seguintes critérios de exclusão: a) sujeitos com menos de seis meses de prática de jump fit ou com frequência mínima de três vezes por semana; b) faixa etária inferior a 40 anos e superior a 50 anos de idade; c) questionário de PAR-Q positivo. Todas foram voluntárias para participar na pesquisa e assinaram o termo de consentimento livre e esclarecido de acordo com a lei 196/96 do Conselho Nacional de Saúde. O presente estudo atendeu às normas para a realização de pesquisas com seres humanos e foi submetido e aprovado pelo Comité de Ética em Pesquisa da Universidade Castelo Branco, Rio de Janeiro, Brasil (protocolo número 056/2008).

\section{Instrumentos e Procedimentos}

Os procedimentos para a realização do estudo envolveram três dias de avaliações que consistiram na recolha de dados, existindo um intervalo de 48 horas entre as mesmas. Na primeira avaliação, foi realizada uma anamnese, aplicado o questionário Par-Q e realizadas as medidas de massa corporal e estatura, através de uma balança de resolução de $0.1 \mathrm{~kg}$ com estadiómetro (Filizola, Brasil). Foi calculada a percentagem de massa gorda estimada através do protocolo de 3 pregas de acordo com Jackson e Pollock (1978). De seguida, as voluntárias foram submetidas a uma familiarização e treino dos protocolos de aula de jump fit e hidro jump com a utilização da Escala de Perceção Subjetiva de Esforço de Borg (1982). Os sujeitos foram aconselhados a não praticar exercícios físicos durante 48 horas antes da realização dos protocolos de intervenção do segundo e terceiro momentos de avaliação.

Nestas visitas os indivíduos foram submetidos a uma sessão de aula de hidro jump e outra de jump fit, com entrada aleatória e contrabalançada e com um intervalo de 48 horas entre as mesmas. A variável $\mathrm{FC}$ foi aferida a cada 2 minutos juntamente com a PSE. O LA foi recolhido a cada 20 minutos. Foram utilizados os minitrampolins (Physicus, Brasil) durante as aulas no solo e na água. Na sessão de jump fit a temperatura ambiente ficou entre $22^{\circ} \mathrm{C}$ e $24^{\circ} \mathrm{C}$ com humidade relativa do ar de $50 \%$. A sessão de aula de hidro jump foi feita em piscina aquecida com profundidade de $1.10 \mathrm{~m}$ e temperatura da água de $28^{\circ} \mathrm{C}$.

Para as análises de sangue os dados foram recolhidos em repouso, 20 minutos e 40 minutos após iniciadas as sessões de treino, do lóbulo do dedo que era aquecido em água com temperaturas entre os $41^{\circ} \mathrm{C}$ e $43^{\circ} \mathrm{C}$ durante 1 a 2 minutos (Pöyhönen, Spilã, Keskinen, Hautala, Savolainene, \& Malkia, 2002), sendo recolhidos $25 \mu \mathrm{l}$ de sangue arterializado, através de capilares de vidro heparinizados e calibrados. O sangue recolhido foi depositado em tubos de $1.5 \mathrm{~mL}$ para microcentrifugas, contendo $50 \mu \mathrm{l}$ de fluoreto de sódio ( $\mathrm{NaF}-1 \%$ ), para desta forma se obter a concentração de lactato sanguíneo $(\mathrm{mM})$ em lactímetro Eletroquímico Yellow Spings Instruments (YSI) modelo 1500 Sport (Roseguini, Silva, \& Gobatto, 2008).

As avaliações da FC durante os protocolos de intervenção foram realizadas de forma contínua e no repouso foram realizadas no meio terrestre e aquático, através de um frequencímetro da marca Polar (Modelo A1, Finlândia).

Os sujeitos permaneceram sentados, numa sala isolada, no máximo repouso possível durante 10 minutos. Logo após, foi feita a recolha com os sujeitos em pé no solo, por dois minutos, e em pé em imersão até à profundidade do apêndice xifóide, também por dois minutos (Freire \& Novaes, 2005).

O protocolo de intervenção utilizado foi o mesmo protocolo nas aulas de jump no solo e na água, com duração de 40 minutos. A aula foi composta do $1^{\circ}$ ao $5^{\circ}$ minuto pelo aquecimento; do $5^{\circ}$ ao $35^{\circ}$ minuto pela parte principal, utilizando-se $140 \mathrm{~b}$ pm de estímulo musical; e do $35^{\circ}$ ao $40^{\circ}$ minuto pelo retorno à calma. Os exercícios executados em cada fase podem ser observados na Tabela 1. 
Tabela 1

Descrição do protocolo de aula de jump com as fases, o tempo de duração, os movimentos executados e os materiais utilizados

\begin{tabular}{|c|c|c|c|}
\hline Fases & Tempo & Movimento executado & Material utilizado \\
\hline Aquecimento & $1^{\circ}$ ao $4^{\circ}$ & $\begin{array}{l}\text { Aquecimento/alongamento das articulações e } \\
\text { da musculatura corporal }\end{array}$ & Nenhum \\
\hline Fase 1 & $5^{\circ}$ ao $10^{\circ}$ & $\begin{array}{l}\text { Corrida }+ \text { chute a frente }+ \text { corrida }+ \text { chute a } \\
\text { frente }+ \text { corrida }+ \text { tesoura frontal }\end{array}$ & Minitrampolim \\
\hline Fase 2 & $11^{\circ}$ ao $16^{\circ}$ & $\begin{array}{l}\text { Corrida }+ \text { chute a frente }+ \text { corrida }+ \text { tesoura } \\
\text { frontal }+ \text { corrida }+ \text { chute lateral }\end{array}$ & Minitrampolim \\
\hline Fase 3 & $17^{\circ}$ ao $22^{\circ}$ & $\begin{array}{c}\text { Corrida }+ \text { chute a frente }+ \text { corrida }+ \text { tesoura } \\
\text { Frontal }+ \text { corrida }+ \text { chute lateral }\end{array}$ & Minitrampolim \\
\hline Fase 4 & $23^{\circ}$ ao $28^{\circ}$ & $\begin{array}{l}\text { Corrida }+ \text { tesoura lateral }+ \text { corrida }+ \text { chute a } \\
\quad \text { frente }+ \text { corrida }+ \text { tesoura frontal }\end{array}$ & Minitrampolim \\
\hline Fase 5 & $29^{\circ}$ ao $34^{\circ}$ & $\begin{array}{l}\text { Corrida }+ \text { chute lateral }+ \text { corrida }+ \text { tesoura } \\
\text { lateral }+ \text { corrida }+ \text { elevação de joelhos }\end{array}$ & Minitrampolim \\
\hline Recuperação & $35^{\circ}$ ao $40^{\circ}$ & $\begin{array}{l}\text { Volta à calma com exercícios de alongamento } \\
\text { e soltura }\end{array}$ & Nenhum \\
\hline
\end{tabular}

\section{Análise Estatística}

O tratamento estatístico dos dados foi realizado através do programa SPSS 14.0 e estes foram apresentados com média e desvio padrão. O teste de Shapiro-Wilk foi usado para verificar a homogeneidade da amostra e o teste de Levene foi aplicado para analisar a homogeneidade de variância. A análise de variância (ANOVA one-way), seguida do Post Hoc de Tukey, foi utilizada para comparar as diferenças de médias por estágio entre as aulas de jump fit e hidro jump nas variáveis de FC e LA. O teste de Friedman, seguido do teste Wilcoxon, foi utilizado para comparar diferenças entre as médias da PSE. O estudo admitiu um nível de $p<.05$ para a significância estatística.

\section{RESULTADOS}

As figuras a seguir apresentam a relação entre as cinco fases específicas, durante as aulas de jump fit e hidro jump, com as variáveis dependentes que foram medidas.

Na Figura 1, observa-se que não houve diferenças significativas nos valores das médias da FC entre as fases dos protocolos das aulas de hidro jump e jump fit $(p<.05)$. Pode observar-se que os maiores valores de FC ocorreram na fase 2 e 5 para o jump e na fase 2 para o hidro jump.
$\mathrm{Na}$ Figura 2, à medida que a atividade aumentava, o tempo de duração em resposta tinha uma PSE mais elevada. Comparando os métodos, para os valores médios da PSE, verifica-se que não houve diferenças significativas entre as fases dos protocolos das aulas de hidro jump e jump fit $(p<.05)$. Entretanto, em todas as fases a PSE apresentou maiores pontuações no protocolo de aula de hidro jump.

$\mathrm{Na}$ Figura 3, verifica-se que não houve diferenças significativas nos valores das médias do LA entre os distintos tempos recolhidos do protocolo de hidro jump quando comparados com os valores do protocolo de jump fit. Entretanto, as diferenças significativas intra-grupos só ocorreram quando foram comparados os tempos de 20 e 40 min com a medida realizada em repouso.

\section{DISCUSSÃO}

O objetivo deste estudo foi comparar os efeitos agudos da prática do jump na frequência cardíaca (FC), nos níveis de lactato sanguíneo (LA) e na perceção subjetiva de esforço (PSE) num mesmo protocolo de aula em meio aquático e terrestre. Os resultados demonstraram não haver diferenças significativas para as variáveis investigadas, entre os métodos jump fit e hidro jump. Entretanto, os dois métodos 
56 | H.B. Moraes, R.G. Vale, A.L. Gomes, G.S. Novaes, J.V. Alves, D.A. Marinho, J.S. Novaes

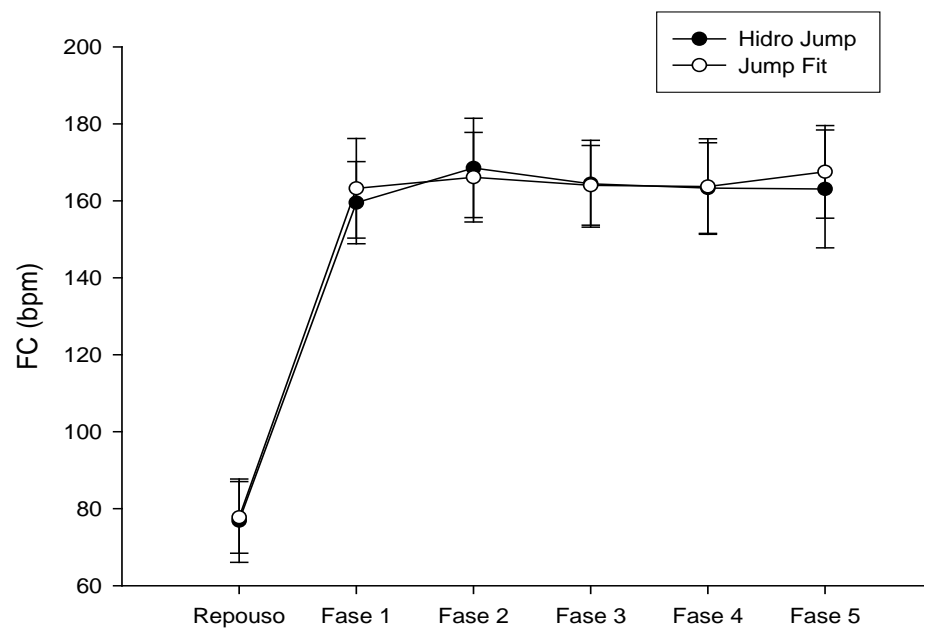

Figura 1. Comparação da frequência cardíaca do hidro jump vs jump fit

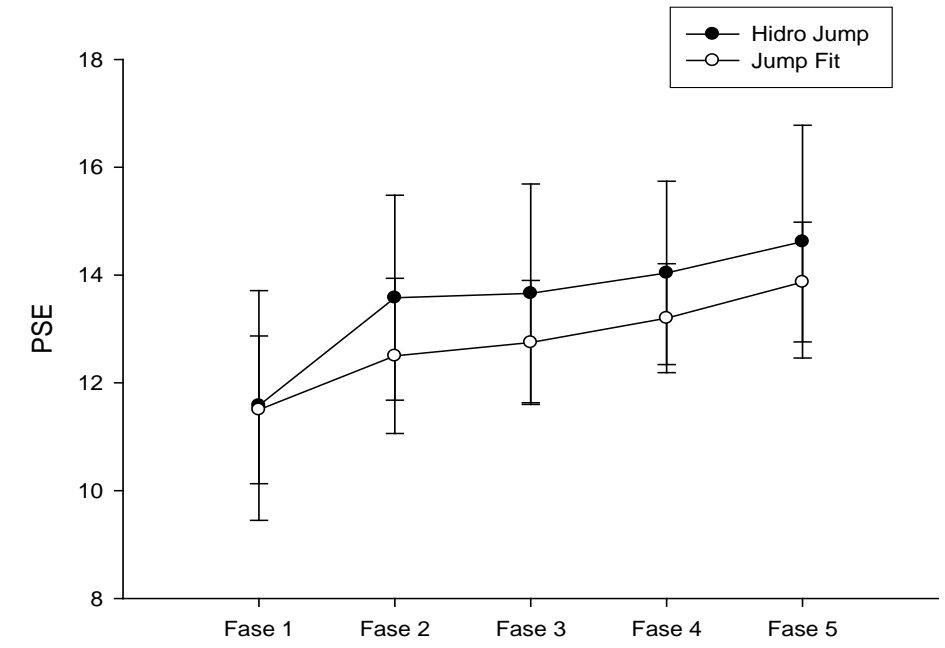

Figura 2. Comparação da percepção subjetiva do esforço do hidro jump vs jump fit

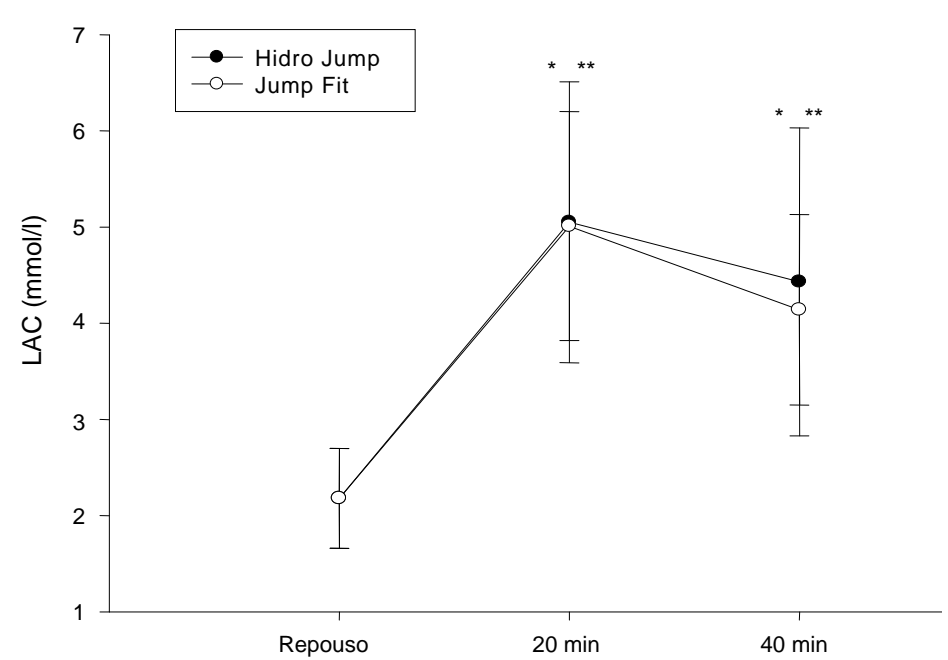

Figura 3. Comparação na concentração plasmática do lactato inter-grupo e intra-grupo do hidro jump e do jump fit, ao longo do tempo de aula (Nota: ${ }^{*} p<.05$, repouso vs 20 min, repouso vs 40 min hidro jump; ** $p<.05$, repouso vs $20 \mathrm{~min}$, repouso vs $40 \mathrm{~min}$ jump fit) 
apresentaram um comportamento de stress cardíaco de acordo com as linhas orientadoras propostas pelo ACSM (American College of Sports Medicine, 2006).

Furtado et al. (2004) verificaram o efeito agudo das variáveis frequência cardíaca (FC), consumo de oxigénio $\left(\mathrm{VO}_{2}\right)$, produção de dióxido de carbono $\left(\mathrm{VCO}_{2}\right)$, razão de troca respiratória (R), equivalente metabólico (MET) e gasto energético, durante uma aula de jump fit, em 10 mulheres praticantes dessa atividade. A avaliação espirométrica das diversas etapas da aula indicou os seguintes resultados médios: FC de $16.3 \pm 8.9 \mathrm{bpm}, \mathrm{VO}_{2}$ de $1.59 \mathrm{~L} \cdot \mathrm{min}^{-1} \pm$ 0.45 , $\mathrm{R}$ de $0.87 \pm 0.10$ e gasto energético total $386.4 \pm 13.8 \mathrm{kcal}$. A intensidade média da aula de jump fit correspondeu a $75 \pm 7.7 \%$ do pico de $\mathrm{VO}_{2}$. Os valores médios percentuais encontrados foram para a $\mathrm{FC}$ de $87.1 \%$ e para o $\mathrm{VO}_{2}$ de $81.2 \%$ ). Num outro estudo, Groosl, Guglielmo, e Carminatti (2008) determinaram a intensidade da aula de power jump por meio da FC. Além do teste incremental, a amostra teve a FC monitorada em dois diferentes protocolos de aulas de jump. Os resultados da FC obtidos durante os dois protocolos, relacionando com a $\mathrm{FC}_{\text {máx }}$, identificou um valor percentual de 82.8 $\pm 6.0 \%$ e $80.0 \pm 5.0 \%$, respetivamente.

Mais recentemente, Perantoni et al. (2009) desenvolveram uma investigação que teve como objetivo verificar a intensidade de uma sessão de jump, realizada com uma cadência musical de 135 batidas por minuto (bpm) acompanhada de uma coreografia somente de membros inferiores. A amostra foi constituída por 11 mulheres saudáveis praticantes da modalidade jump. As voluntárias foram submetidas a um teste no minitrampolim para verificar o consumo de oxigénio $\left(\mathrm{VO}_{2}\right)$ e a frequência cardíaca (FC) durante a realização de uma coreografia com 10 minutos. Encontrou-se um percentual médio para a $\mathrm{FC}$ de $81 \%$ e para o $\mathrm{VO}_{2}$ de $64 \%$. Os resultados de todas as pesquisas apresentadas anteriormente demonstraram ser similares aos resultados do presente estudo, visto que as respostas de FC mantiveram-se elevadas, acima dos $80 \%$ da $\mathrm{FC}_{\text {máx }}$ predito pela idade (American College of Sports Medicine, 2006).

Leite, Alonso, Anjos, Gonçalves e Padovani (2006) verificaram o impacto de 16 semanas de treino de hidro jump no comportamento das variáveis da aptidão física, em mulheres sedentárias. A amostra foi composta por 20 mulheres sedentárias, com idades entre os 20 e os 35 anos. O treino foi composto por três sessões semanais de 45 minutos com o minitrampolim na água. Os resultados indicaram aumentos significativos da resistência muscular de membros inferiores e superiores, da frequência cardíaca pós-exercício e do consumo máximo de oxigênio. Sendo assim, um treino com minitrampolim na água é eficaz para incremento crónico de algumas capacidades físicas relacionadas com indicadores de saúde.

Os resultados do presente estudo (referentes aos valores de frequência cardíaca) demonstraram um stress agudo favorável das variáveis fisiológicas no jump em meio aquático e terrestre, o que é corroborado com os resultados da pesquisa anterior. Neste âmbito, Caromano, Themudo Filho, e Candeloro, (2003) advertem que um treino sistematizado, dentro dos padrões determinados pelos consensos da Revista Brasileira de Medicina do Esporte, (2001) e do ACSM (2006), executado por um indivíduo em imersão no meio líquido, pode levar o organismo a novas adaptações cardiovasculares. Isto poderá acontecer em virtude de uma série de modificações fisiológicas no fluxo de sangue, na termorregulação, no metabolismo, no sistema nervoso, na composição sanguínea, na secreção das glândulas e nos aspetos psicofisiológicos.

Anjos et al. (2006) compararam o efeito do treino de um jump em solo e em meio aquático durante 16 semanas nas dimensões morfológicas (índice de massa corporal - IMC, relação cintura-quadril - RCQ) e na capacidade cardiorespiratória $\left(\mathrm{VO}_{2 \operatorname{máx}}\right)$ de 46 mulheres sedentárias, entre os 20 e os 35 anos. Os resultados indicaram uma manutenção da massa corporal e a redução não significativa do IMC em ambos os grupos. Porém, houve uma redução dos 
valores da RCQ apenas para o grupo de hidro jump, com um incremento da capacidade cardiorrespiratória de $22 \%$ para a atividade de solo e de $9 \%$ para a aquática. Apesar de terem ocorrido melhorias na capacidade aeróbia nas duas propostas, esta foi maior no exercício em solo, enquanto no hidro jump se notou uma maior influência sobre as outras variáveis morfológicas do estudo. Isto aconteceu, provavelmente, em virtude do tipo de exigência imposta pelos dois protocolos. Entretanto, quando se observam os valores da média da FC de recuperação, no início e no final da intervenção, do estudo acima descrito, os valores foram significativamente maiores no meio aquático, quando comparados com os do meio terrestre.

No estudo de Ferreira, Brasil, Barreto, Dos Santos, Vale e Novaes, (2005), as médias dos valores da FC na sessão de ciclismo indoor também foram superiores do que as do ciclismo aquático. No nosso estudo, apesar de ter sido realizado com o minitrampolim, o mesmo comportamento fisiológico aconteceu apenas na fase 2. Ou seja, a FC no meio terrestre esteve mais elevada do que no meio aquático. Isto contrariou a hipótese inicial do nosso estudo, uma vez que se esperava que durante a comparação da atividade jump, dentro e fora da água, a FC fosse menor no meio líquido, já que a pressão hidrostática e a flutuação do corpo, dada pela força de impulsão da água, auxiliam o deslocamento sanguíneo para a região central do corpo, aumentando o volume sistólico e diminuindo a FC. Um dos estudos que sustentou a nossa hipótese foi o de Graef e Kruel (2006), que numa revisão sobre o comportamento da FC e da PSE nos meios aquático e terrestre, sugerem haver uma tendência para maiores diferenças entre estas quando os indivíduos se aproximam do esforço máximo, apresentando uma redução da FC em ambiente aquático.

Rennie, Di Prampero e Cerretelli (1991), corroborando com os dados da presente pesquisa, não revelaram alterações significativas na FC para exercícios intensos, em temperaturas de água abaixo de $34^{\circ} \mathrm{C}$. No entanto,
Kruel (2000) comparou a FC durante a execução de exercícios típicos de hidroginástica realizados dentro e fora do ambiente aquático. Os resultados indicaram uma redução média de $25 \mathrm{bpm}(p<.05)$, durante a realização de exercícios na água com intensidade moderada, para mulheres praticantes de hidroginástica há pelo menos seis meses. Entretanto, no nosso estudo, a amostra era constituída por praticantes de actividade terrestre de jump fit sem experiência no hidro jump. Sabe-se que a falta de um período de adaptação ao meio líquido pode influenciar as respostas hemodinâmicas (Leite et al., 2006). Este facto, e o tamanho reduzido da amostra, podem ser assumidos como uma limitação do nosso estudo.

Em relação à PSE, no nosso estudo, quando comparados os valores médios dos dois métodos, verificou-se que não houve diferenças significativas entre as fases dos protocolos das aulas dentro e fora da água. Entretanto, em todas as fases, a PSE apresentou maiores pontuações no protocolo de aula de hidro jump. Estes resultados estão de acordo com a revisão de Graef e Kruel (2006), que advertem que a sensação de cansaço percebida pelo praticante, numa atividade realizada no meio aquático, é sempre maior que no meio terrestre. Contudo, parece não haver um consenso na literatura a respeito da relação entre FC e PSE no meio aquático, apontando para a realização de novas investigações neste âmbito.

Perantoni et al. (2009) verificaram que a PSE média de uma sessão de jump training foi de $12.4 \pm 2.3$, a partir da escala de percepção de esforço de Borg. Estes resultados são semelhantes aos encontrados na presente pesquisa onde a PSE média da aula de jump fit variou entre o nível de intensidade 11 da escala de Borg, na fase 1 da aula, e o nível 13, na fase 5 . Lazzari e Meyer (1997), compararam as respostas da PSE durante a caminhada na água, com a água pela cintura, com a caminhada na passadeira a uma mesma velocidade, com intensidade submáxima. Os valores da PSE recolhidos na caminhada aquática foram significativamente maiores quando comparados com os 
valores da caminhada na passadeira. Estes resultados corroboram os nossos e a nossa hipótese em relação à PSE. Esta diferença, em ambos os ambientes, já era esperada em virtude da utilização da mesma velocidade para os movimentos no minitrampolim, controlada pelo ritmo musical (140 bpm). Sabe-se que o meio aquático apresenta maior resistência ao deslocamento do corpo causando um maior gasto energético e trabalho muscular (Graef \& Kruel, 2006).

O estudo de Ferreira et al. (2005) também comparou a variável PSE nas fases do protocolo de aula de ciclismo indoor e aquático. Os resultados apresentaram valores semelhantes aos do presente estudo. Ou seja, um aumento da sensação de esforço a cada fase em ambos os protocolos. Este facto ocorre principalmente por o volume ser uma variável capaz de exercer um elevado desgaste em diferentes sessões de treino (Bassini-Cameron, Monteiro, Gomes, Werneck-de-Castro, \& Cameron, 2008). Ainda no estudo de Ferreira et al. (2005), os indivíduos apresentaram valores maiores da PSE no ciclismo aquático, nas várias fases da parte principal da aula, quando comparado com o terrestre, porém apresentando diferenças significativas $(p<.05)$ apenas na fase 2 . Esta situação ocorreu possivelmente pela maior fadiga periférica proporcionada pelo meio aquático que pode levar o indivíduo a uma maior sensação de esforço, independentemente do tipo de exercícios executado, corroborando desta forma os resultados do nosso estudo com os do estudo de Di Masi et al. (2007).

O valor médio da variável LA foi maior na sessão de hidro jump em todos os tempos de verificação, embora estas diferenças não se tenham verificado estatisticamente significativas. Este facto ocorreu também no estudo de Ferreira et al. (2005), quando foi comparada a concentração do lactato sanguíneo entre um protocolo de aula de spinning dentro e fora de água. Os resultados indicaram pouca variação das respostas do lactato em meio aquático e terrestre $(p>.05)$, indo de encontro aos resultados obtidos por Di Masi et al. (2007), os quais analisaram a concentração de lactato durante a recuperação nos dois meios. Tanto num protocolo de esforço, como num protocolo de recuperação, a pequena alteração de lactato, verificada entre os dois diferentes meios, aponta para um índice mais elevado em meio aquático. Esta situação acontece, possivelmente pelo aumento da fadiga periférica do indivíduo que resulta de uma maior resistência oferecida pela água para a realização do movimento.

As diferenças significativas encontradas no $20^{\circ}$ e no $40^{\circ}$ minutos da aula, quando comparados ao repouso, nos meios aquático e terrestre, são decorrentes de um mecanismo que é proposto como uma forma de alimentação das exigências energéticas dos diferentes métodos de exercício físico (Bassini-Cameron et al., 2008; Di Masi et al., 2007). Pode notar-se que a concentração de lactato no $20^{\circ}$ minuto foi de $5 \mathrm{mM} / \mathrm{L}$ para ambos os métodos e no $40^{\circ}$ minuto a média dos dois métodos foi de 4.3 $\mathrm{mM} / \mathrm{L}$. Tais respostas metabólicas demonstram que o método jump não é excessivo nem dentro nem fora da água, pois as intensidades de trabalho correspondem a valores ligeiramente acima ou abaixo do limiar anaeróbio. Esta forma de treino que propõe um estímulo contínuo resulta num aumento do condicionamento físico e desta forma, pode proporcionar benefícios à saúde (Mello, Boscolo, Esteves, \& Tukik, 2005; Okano et al., 2006; Pinto, Alberton, Figueiredo, Tiggemann, \& Kruel, 2008).

\section{CONCLUSÕES}

A partir dos resultados encontrados e assumindo a limitação de uma amostra reduzida, o presente estudo revelou não haver diferenças significativas entre as variáveis $\mathrm{FC}$, a PSE e o LA durante os protocolos de aulas de jump no meio aquático e terrestre. Como aplicação prática pode-se sugerir estas actividades para indivíduos que pretendem obter melhorias do condicionamento físico geral, podendo utilizar-se o exercício dentro e fora de água.

\section{Agradecimentos:}

Nada a declarar. 
Conflito de Interesses:

Nada a declarar.

Financiamento:

Nada a declarar.

\section{REFERÊNCIAS}

Alonso, P. T., Anjos, T. C., Leite, J. P, Gonçalves, A., \& Padovani, C. (2005). Composição corporal, aptidão física e qualidade de vida em mulheres jovens em exercícios no mini-trampolim. Arquivos em Movimento, 1(2), 49-58.

American College of Sports Medicine (2006). ACSM's guidelines for exercise testing and prescription ( $7^{\text {a }}$ ed.). Philadelphia: Lippincott Williams \& Wilkins.

Anjos, T. C., Leite, J. P., Alonso, P. T., Gonçalves, A., \& Padovani, C. (2006). Variáveis de condicionamento físico relacionado à saúde em adultas jovens submetidas a dois programas de atividade física: Rebound exercise em solo e água. Fitness \& Performance Journal, 5(1), 17-22.

Anjos, T. C., Leite, J. P., Alonso, P. T., Gonçalves, A., \& Padovani, C. (2007). Composição corporal em mulheres jovens em treinamento em mini-trampolim em solo e em água com 16 semanas de duração. Arquivos em Movimento, 3(1), 39-50.

Bassini-Cameron, A., Monteiro, A. N., Gomes, A. L., Werneck-de-Castro, J. P., \& Cameron L. C. (2008). Glutamine protects against increases in blood ammonia in football players in an exercise intensity dependent way. British Journal of Sports Medicine, 42, 260-266. doi: 10.1136/bj sm.2007.040378

Borg, G. (1982). Psychophysical bases of perceived exertion. Medicine $\mathcal{E}$ Science in Sports $\mathcal{E}$ Exercise, 14, 377-381.

Caromano, F. A., Themudo Filho, M. R., \& Candeloro, J. M. (2003). Efeitos fisiológicos da imersão e do exercício na água. Fisiologia Brasil, 4(1), 61-66.

Di Masi, F., Vale, R. G., Dantas, E. H., Barreto, A. C., Novaes, J. S., \& Reis, V. M. (2007). Is blood lactate removal during half-liquid cycling faster than during dry land cycling? Journal of Sports Science and Medicine, 6, 188-192.

Ferreira, A. C., Brasil, R. M., Barreto, A. N., Dos Santos, M. A., Vale, R. G., \& Novaes, J. S. (2005). Comparação das respostas hemodinâ- micas entre o ciclismo indoor e aquático. Arquivos em Movimento, 1 (2), 29-38.

Freire, V. B., \& Novaes, J. S. (2005). Comparison between heart rate responses for the same exercise with and without aquatic jump equipment. Aquatic Fitness Research Journal, 2(1), 36-43.

Furtado, E. S., Simão, R., \& Lemos, A. L. (2004). Análise do consumo de oxigênio, freqüência cardíaca e dispêndio energético, durante as aulas do Jump Fit. Revista Brasileira de Medicina do Esporte, 10(5), 371-375. doi: 10.1590/S151786922004000500004

Graef, F. I., \& Kruel, L. F. (2006). Freqüência cardíaca e percepção subjetiva do esforço no meio aquático: Diferenças em relação ao meio terrestre e aplicações na prescrição do exercício: Uma revisão. Revista Brasileira de Medicina do Esporte, 12(4), 221-228. doi: 10.1590/S 1517-8692206000400011

Grossl T., Gugliemo, L. G., Carminatti, L. J., \& Silva, J. F. (2008). Determinação da intensidade da aula de power jump por meio da freqüência cardíaca. Revista Brasileira de Cineantropometria e Desempenho Humano, 10(2), 129-136.

Jackson, A. S., \& Pollock, M. L. (1978). Generalized equations for predicting body density of men. British Journal of Nutricional, 4, 497-504.

Kruel, L. F. (2000). Alterações fisiológicas e biomecânicas em indivíduos praticando exercícios de hidroginástica dentro e fora d'àgua (Tese de doutorado não publicada). Universidade Santa Maria, Rio Grande do Sul, Brasil.

Kruel, L. F., Tartaruga, L. A., Dias, A. C., Silva, R. C., Picanço, P. S., \& Rangel, A. B. (2005). Heart hate during water immersion. Aquatic Fitness Research Journal, 2(1), 7-13.

Lazzari, J. M., \& Meyer, F. (1997). Freqüência cardíaca e percepção do esforço na caminhada aquática e na esteira em mulheres sedentárias e com diferentes percentuais de gordura. Revista Brasileira de Atividade Física e Saúde, 3, 7-13.

Leite, J. L., Alonso, P. T., Anjos, T. C., Gonçalves, A., \& Padovani, C. R. (2006). Efeitos adaptativos do treinamento com mini-trampolim dentro da água. Revista Brasileira de Ciência e Movimento, 14(3), 23-28.

Lemos, A., Simão R., Miranda, H., \& Novaes, J.S. (2007). A influência aguda de uma aula de mini-trampolim no agachamento. Fitness $\mathcal{E}$ Performance Journal, 6(2), 76-81. doi:10.3900/fpj.6. 2.76.p 
Lemos, A., Simão, R., Miranda, H., \& Novaes, J. S. (2008). A influência aguda de uma sessão de jump fit no desempenho dos exercícios resistidos. Revista Brasileira de Fisiologia do Exercício, 7(3), 118-122.

Mello, M. T., Boscolo, R. A., Esteves, A., \& Tukik, S. (2005). O exercício físico e os aspectos psicobiológicos. Revista Brasileira de Medicina do Esporte, 11 (3), 203-207.

Okano, A. H., Altimari, L. R., Simões, H. G., De Moraes, A. C., Nakamura, F. Y., Cyrino, E. S., \& Burini, R. C. (2006). Comparação entre limiar anaeróbio determinado por variáveis ventilatórias e pela resposta do lactato sanguíneo em ciclistas. Revista Brasileira de Medicina do Esporte, 12(1), 39-44. doi: 10.1590/S1517-869220060 00100008

Peratoni, C.B., Derez, C.S., Lauria, A.A., Lima, J.P., \& Novaes, J.S. (2009). Análise da intensidade de uma sessão de jump training. Fitness $\mathcal{E}$ Performance Journal, 8(4), 286-290. doi: 10.390 0/fpj.8.4.286.p

Pinto, S. S., Alberton, C. L., Figueiredo, P. A., Tiggemann, C. L., \& Kruel, L. F. (2008). Respostas de freqüência cardíaca, consumo de oxigênio e sensação subjetiva ao esforço em um exercício de hidroginástica executado por mulheres em diferentes situações com e sem o equipamento de aquafins. Revista Brasileira de Medicina do Esporte, 4(4), 357-361. doi: 10.159 0/S1517-86922008000400007

Poyhonen, T., Sipilã, S., Keskinen, K. L., Hautala, A., Savolainen, J., \& Malkia, E. (2002). Effects of aquatic resistance training on neuromuscular performance in healthy women. Medicine $\mathcal{E}$ Science in Sports \& Exercise, 34(12), 2103-2109.

Rennie, D. W., Di Prampero, P., \& Cerretelli, P. (1991). Effects of water immersion on cardiac output, heart rate and stroke volume of man at rest and during exercise. Journal of Sports Medicine, 24, 223-228.

Revista Brasileira de Medicina do Esporte (2001). Posicionamento oficial da Sociedade Brasileira de Medicina do Esporte sobre Esporte competitivo em Indivíduos acima de 35 anos. Revista Brasileira de Medicina do Esporte, 7(3), 8392. doi: 10.1590/S1517-86922001000300004

Roseguini, A. Z., Da Silva, A. S., \& Gobatto, C. A. (2008). Determinações e relações dos parâmetros anaeróbios do RAST, do limiar anaeróbio e da resposta lactacidemica obtida no inicio, no intervalo e ao final de uma partida oficial de handebol. Revista Brasileira de Medicina do Esporte, 14(1), 46-50.

Smith, J. F. \& Bishop, P. A. (1998). Rebounding exercise: Are the training effects sufficient for cardiorespiratory fitness? Journal of Sports Medicine, 5(1), 6-10.

Svedenhag, J., \& Seger, J. (1992). Running on land and in water: Comparative exercise physiology. Medicine \& Science in Sports \& Exercise, 10, 11551160.

Vendrusculo, A. P., Tartaruga, L. A., Coertjens, M., Pantoja, P. D., Petkowicz, D., \& Kruel, L. F. (2004). Sensação subjetiva ao esforço e consumo máximo de oxigênio. Revista Brasileira de Fisiologia do Exercício, 1(1), 128-134.

(c)) EY-Nc Todo o conteúdo da revista Motricidade está licenciado sob a Creative Commons, exceto quando especificado em contrário e nos conteúdos retirados de outras fontes bibliográficas. 